\title{
An Effective Algorithm for Globally Solving Sum of Linear Ratios Problems
}

\author{
Hongwei Jiao, ${ }^{1}$ Lei Cai, ${ }^{2}$ Zhisong Hou, ${ }^{2}$ and Chunyang Bai ${ }^{1}$ \\ ${ }^{1}$ School of Mathematical Sciences, Henan Institute of Science and Technology, Xinxiang 453003, China \\ ${ }^{2}$ School of Information Engineering, Henan Institute of Science and Technology, Xinxiang 453003, China \\ Correspondence should be addressed to Hongwei Jiao; jiaohongwei@126.com and Lei Cai; cailei2014@126.com
}

Received 21 February 2017; Accepted 13 April 2017; Published 5 June 2017

Academic Editor: Haibo Du

Copyright (C) 2017 Hongwei Jiao et al. This is an open access article distributed under the Creative Commons Attribution License, which permits unrestricted use, distribution, and reproduction in any medium, provided the original work is properly cited.

In this study, we propose an effective algorithm for globally solving the sum of linear ratios problems. Firstly, by introducing new variables, we transform the initial problem into an equivalent nonconvex programming problem. Secondly, by utilizing direct relaxation, the linear relaxation programming problem of the equivalent problem can be constructed. Thirdly, in order to improve the computational efficiency of the algorithm, an out space pruning technique is derived, which offers a possibility of pruning a large part of the out space region which does not contain the optimal solution of the equivalent problem. Fourthly, based on out space partition, by combining bounding technique and pruning technique, a new out space branch-and-bound algorithm for globally solving the sum of linear ratios problems (SLRP) is designed. Finally, numerical experimental results are presented to demonstrate both computational efficiency and solution quality of the proposed algorithm.

\section{Introduction}

Sum of linear ratios problems (SLRP) have broad wide applications in information technology, control science and engineering, transportation design, government plan, economy and finance [1-5], and so on. In these applications, especially, the number of linear ratios is usually less than four or five. In addition, due to the fact that the sum of linear ratios problems (SLRP) possess generally multiple local optimal solutions that are not globally optimal, the kinds of problems pose significant theoretical difficulty and computational complexity. Therefore, they have attracted interest of many researchers and practitioners.

In this paper, we will investigate the following sum of linear ratios problems:

$$
\begin{aligned}
(\mathrm{SLRP}): & v=\max G(y)=\sum_{i=1}^{p} \frac{\varphi_{i}(y)}{\phi_{i}(y)} \\
\text { s.t. } & y \in \Lambda \triangleq\left\{y \in R^{n} \mid A y \leq b, \quad y \geq 0\right\},
\end{aligned}
$$

where $A \in R^{m \times n}, b \in R^{m}, \varphi_{i}(y)$, and $\phi_{i}(y)$ are all affine functions, $\Lambda$ is a nonempty bounded constraint set, and for any $y \in \Lambda, \phi_{i}(y) \neq 0, i=1,2, \ldots, p$.
During the past two decades, with the assumption that the denominator $\varphi_{i}(y) \geq 0$ and the numerator $\phi_{i}(y)>0$ for any $y \in \Lambda$, many optimization algorithms have been developed for globally solving the sum of linear ratios problems (SLRP), for example, simplex algorithms, image space algorithm, branch-and-bound algorithms, and monotonic algorithm (see [6-17]). Recently, Jiao et al. [18-20] presented three branch-and-bound algorithms for sum of linear ratios problem by constructing different linear relaxation technique; Jiao et al. [21] presented a new two-level relaxation algorithm for generalized linear multiplicative programming with generalized polynomial constraints, which can be also used to globally solve the sum of linear ratios problem (SLRP) investigated in this paper. In addition, several new algorithms [22-25] have been also proposed for solving linear sum-ofratios and nonlinear sum-of-ratios problems.

In this paper, we shall present a new out space branchand-bound algorithm for the sum of linear ratios problems (SLRP) using out space pruning technique. Firstly, we transform the initial problem into the equivalent nonconvex programming problem by introducing new variables. Next, the linear relaxation programming problem of the equivalent problem can be constructed by utilizing direct relaxation. 
Secondly, by making full use of the special structure of the equivalent problem and the branch-and-bound algorithm, an out space pruning technique is derived to improve the computational efficiency of the proposed algorithm, which offers a possibility of pruning a large part of the investigated out space region which does not contain the global optimal solution of the equivalent problem. Thirdly, by combining bounding technique and pruning technique, a new out space branch-and-bound algorithm is designed for globally solving the sum of linear ratios problems (SLR). Finally, numerical experimental results are presented to demonstrate both computational efficiency and solution quality of the proposed algorithm.

The remaining sections of this paper are organized as follows. At first, in Section 2, by introducing new variables the equivalent nonconvex programming problem of the initial problem is introduced. Next, by utilizing direct relaxation the linear relaxation programming problem of the equivalent problem is constructed. Second, an out space pruning technique is derived by making full use of the special structure of the equivalent problem and the branchand-bound algorithm in Section 3. Third, a new out space branch-and-bound algorithm and its global convergence are described in Section 4. Fourth, numerical experiments and their computational results are presented in Section 5. Finally, the concluding remarks of this paper are drawn.

\section{Equivalent Problem and Its Linear Relaxation}

In this section, to globally solve the problem (SLRP), by the continuity of the linear ratio $\varphi_{i}(y) / \phi_{i}(y)$, we know that $\phi_{i}(y)>0$ or $\phi_{i}(y)<0$. Here, without loss of generality, we can assume that

$$
\begin{aligned}
& \phi_{i}(y)>0, \quad i=1,2, \ldots, T ; \\
& \phi_{i}(y)<0, \quad i=T+1, T+2, \ldots, p
\end{aligned}
$$

except for the above assumptions, for each $i \in\{1,2, \ldots, p\}$, since

$$
\frac{\varphi_{i}(y)}{\phi_{i}(y)}=\frac{\varphi_{i}(y)+M_{i} \phi_{i}(y)}{\phi_{i}(y)}-M_{i},
$$

where $M_{i}$ is an appropriate real number such that $\varphi_{i}(y)+$ $M_{i} \phi_{i}(y) \geq 0$. Thus, without loss of generality, by some proper transformation we can always guarantee that the numerator of each ratio is greater than or equal to 0 .

Let

$$
\begin{gathered}
U_{i}^{0}= \begin{cases}\frac{1}{\min _{y \in \Lambda} \phi_{i}(y)}, & i=1,2, \ldots, T, \\
\frac{1}{-\max _{y \in \Lambda} \phi_{i}(y)}, & i=T+1, \ldots, p ;\end{cases} \\
L_{i}^{0}= \begin{cases}\frac{1}{\max _{y \in \Lambda} \phi_{i}(y)}, & i=1,2, \ldots, T, \\
\frac{1}{-\min _{y \in \Lambda} \phi_{i}(y)}, & i=T+1, \ldots, p .\end{cases}
\end{gathered}
$$

And construct the initial box

$$
Z^{0}=\left\{z \in R^{p} \mid L_{i}^{0} \leq z_{i} \leq U_{i}^{0}, i=1,2, \ldots, p\right\} ;
$$

then the problem (SLRP) can be converted into the equivalent problem (EQ) as follows.

$$
\begin{aligned}
\operatorname{EQ}\left(Z^{0}\right): \quad & v\left(Z^{0}\right)=\max H_{0}(y, z) \\
& =\sum_{i=1}^{T} z_{i} \varphi_{i}(y)-\sum_{i=T+1}^{p} z_{i} \varphi_{i}(y) \\
\text { s.t. } \quad & H_{i}(y, z)=z_{i} \phi_{i}(y) \leq 1, \quad i=1,2, \ldots, T, \\
& H_{i}(y, z)=z_{i} \phi_{i}(y) \leq-1, \\
& y \in \Lambda, \quad z \in Z^{0} .
\end{aligned}
$$

The key equivalence theorem for the problem (SLRP) and the $\mathrm{EQ}\left(Z^{0}\right)$ is described as follows.

Theorem 1. If $\left(y^{*}, z_{1}^{*}, \ldots, z_{p}^{*}\right)$ is a global optimum point of the problem $E Q\left(Z^{0}\right)$, then $y^{*}$ is a global optimum point of the problem (SLRP). On the contrary, if $y^{*}$ is a global optimum point of the problem (SLRP), then $\left(y^{*}, z_{1}^{*}, \ldots, z_{p}^{*}\right)$ is a global optimum point of the problem $\mathrm{EQ}\left(Z^{0}\right)$ with $z_{i}^{*}=1 / \phi_{i}\left(y^{*}\right), i=$ $1,2, \ldots, T, z_{i}^{*}=1 /-\phi_{i}\left(y^{*}\right), i=T+1, T+2, \ldots, p$.

Proof. The conclusion can be easily drawn, so the proof is omitted.

From Theorem 1, to solve the problem (SLRP), we may globally solve its equivalent nonconvex programming problem $\mathrm{EQ}\left(Z^{0}\right)$ instead.

For each box $Z^{k}=\left\{z \in R^{p} \mid L_{i}^{k} \leq z_{i} \leq U_{i}^{k}, i=\right.$ $1,2, \ldots, p\} \subseteq Z^{0}$, some notations and functions of this paper are listed as follows:

$$
\begin{aligned}
& H_{0}^{U}(y)=\sum_{i=1}^{T} U_{i}^{k} \varphi_{i}(y)-\sum_{i=T+1}^{p} L_{i}^{k} \varphi_{i}(y) ; \\
& H_{0}^{L}(y)=\sum_{i=1}^{T} L_{i}^{k} \varphi_{i}(y)-\sum_{i=T+1}^{p} U_{i}^{k} \varphi_{i}(y) ; \\
& H_{i}^{U}(y)=U_{i}^{k} \phi_{i}(y), \quad i=1,2, \ldots, p \\
& H_{i}^{L}(y)=L_{i}^{k} \phi_{i}(y), \quad i=1,2, \ldots, p .
\end{aligned}
$$

Theorem 2. For the functions $H_{i}^{U}(y), H_{i}^{L}(y), H_{i}(y, z), i=$ $0,1,2, \ldots, p$, where $y \in \Lambda, z \in Z^{k} \subseteq Z^{0}$, we have the following two conclusions:

(i) $H_{i}^{U}(y) \geq H_{i}(y, z) \geq H_{i}^{L}(y), i=0,1,2, \ldots, p$.

(ii) $\lim _{\left\|U^{k}-L^{k}\right\| \rightarrow 0}\left[H_{i}^{U}(y) \quad-\quad H_{i}(y, z)\right] \quad=$ $\lim _{\left\|U^{k}-L^{k}\right\| \rightarrow 0}\left[H_{i}(y, z)-H_{i}^{L}(y)\right]=0$.

Proof. The proof can be easily followed, therefore here it is omitted. 
From the above Theorem 2, for any $Z^{k} \subseteq Z^{0}$, the corresponding linear relaxation programming problem $\operatorname{LRP}\left(Z^{k}\right)$ of the problem $\mathrm{EQ}\left(Z^{k}\right)$ can be established as follows, which can offer a reliable upper bound for the global optimum of $\mathrm{EQ}\left(Z^{k}\right)$.

$$
\begin{aligned}
\operatorname{LRP}\left(Z^{k}\right): \quad & \mathrm{UB}\left(Z^{k}\right)=\max H_{0}^{U}(y) \\
& =\sum_{i=1}^{T} U_{i}^{k} \varphi_{i}(y)-\sum_{i=T+1}^{p} L_{i}^{k} \varphi_{i}(y) \\
\text { s.t. } \quad & H_{i}^{L}(y)=L_{i}^{k} \phi_{i}(y) \leq 1, \quad i=1,2, \ldots, T, \\
& H_{i}^{L}(y)=L_{i}^{k} \phi_{i}(y) \leq-1, \\
& i=T+1, T+2, \ldots, p,
\end{aligned}
$$

$y \in \Lambda$.

\section{Out Space Pruning Technique}

For any box $Z^{k} \subseteq Z^{0}$, we need to recognize whether or not $Z^{k}$ contains global optimum point of the problem (EQ). Thus, in this section, we shall construct an out space pruning technique for pruning a part of the investigated out space region which does not contain the global optimum point of the problem (SLRP) and use the technique to improve the computational efficiency of the proposed algorithm. The proposed new out space pruning technique aims at replacing the box $Z^{k}=\left[L^{k}, U^{k}\right]$ with a smaller box $Z^{\prime}=\left[L^{\prime}, U^{\prime}\right]$ without pruning any global optimum point of the problem (EQ).

We assume, without loss of generality, that LB is a currently known lower bound of the global optimum value of the $\mathrm{EQ}\left(Z^{0}\right)$ and that $v\left(Z^{k}\right)$ denote the maximum value of the problem $H_{0}(y, z)$ over $Z^{k}$ and $D$ and set

$$
\begin{aligned}
l_{i}^{0} & =\min _{y \in \Lambda} \varphi_{i}(y), \quad i=1,2, \ldots, p, \\
u_{i}^{0} & =\max _{y \in \Lambda} \varphi_{i}(y), \quad i=1,2, \ldots, p, \\
\mathrm{RUB}^{k} & =\sum_{i=1}^{T} U_{i}^{k} u_{i}^{0}-\sum_{i=T+1}^{p} L_{i}^{k} l_{i}^{0}, \\
\rho_{i}^{k} & =\frac{\mathrm{LB}-\mathrm{RUB}^{k}+U_{i}^{k} u_{i}^{0}}{u_{i}^{0}}, \quad i=1,2, \ldots, T, \\
\rho_{i}^{k} & =\frac{-\mathrm{LB}+\mathrm{RUB}^{k}+L_{i}^{k} l_{i}^{0}}{l_{i}^{0}}, \quad \\
& \quad i=T+1, T+2, \ldots, p .
\end{aligned}
$$

Theorem 3. For any subbox $Z^{k}=\left(Z_{i}^{k}\right)_{p \times 1}=\left[L_{i}^{k}, U_{i}^{k}\right]_{p \times 1} \subseteq$ $Z^{0}$, we have the following two conclusions:

(i) Suppose that $R U B^{k}<L B$; then there does not exist any global optimum point of $E Q\left(Z^{k}\right)$ over $Z^{k}$. (ii) Suppose that $R U B^{k} \geq L B$; then, when $j \in\{1,2, \ldots, T\}$, there does not exist any global optimum point of $E Q\left(Z^{0}\right)$ over $Z_{a}^{k}$; and when $j \in\{T+1, T+2, \ldots, p\}$, there does not exist any global optimum point of $E Q\left(Z^{0}\right)$ over $Z_{b}^{k}$, where

$$
\begin{aligned}
& Z_{a}^{k}=\left(Z_{a i}^{k}\right)_{p \times 1} \subseteq Z^{0} \\
& \text { with } Z_{a i}^{k}= \begin{cases}Z_{i}^{k}, & i \neq j, i=1,2, \ldots, p, \\
{\left[L_{i}^{k}, \rho_{i}^{k}\right) \cap Z_{i}^{k},} & i=j \in\{1,2, \ldots, T\} .\end{cases} \\
& Z_{b}^{k}=\left(Z_{b i}^{k}\right)_{p \times 1} \subseteq Z^{0} \\
& \text { with } Z_{b i}^{k}= \begin{cases}Z_{i}^{k}, & i \neq j, i=1,2, \ldots, p, \\
\left(\rho_{i}^{k}, U_{i}^{k}\right] \cap Z_{i}^{k}, & i=j \in\{T+1, T+2, \ldots, p\},\end{cases}
\end{aligned}
$$

Proof. (i) Suppose that $\mathrm{RUB}^{k}<\mathrm{LB}$; then we have

$$
\begin{aligned}
v\left(Z^{k}\right) & =\max _{z \in Z^{k}, y \in \Lambda} \sum_{i=1}^{p} z_{i} \varphi_{i}(y) \leq \sum_{i=1}^{p} \max _{z \in Z^{k}, y \in \Lambda} z_{i} \varphi_{i}(y) \\
& =\sum_{i=1}^{T} U_{i}^{k} u_{i}^{0}-\sum_{i=T+1}^{p} L_{i}^{k} l_{i}^{0}=\mathrm{RUB}^{k}<\mathrm{LB} ;
\end{aligned}
$$

therefore, there does not exist any global optimum point of the problem $\mathrm{EQ}\left(Z^{k}\right)$ over $Z^{k}$.

(ii) Suppose that $\mathrm{RUB}^{k} \geq \mathrm{LB}$; then we can get the following several conclusions.

When $j \in\{1,2, \ldots, T\}$, for any $y \in \Lambda$ and $z \in Z_{a}^{k}$, since

$$
\begin{aligned}
& 0 \leq l_{i}^{0} \leq \varphi_{i}(y) \leq u_{i}^{0}, \\
& 0 \leq L_{i}^{k} \leq z_{i} \leq U_{i}^{k}, \\
& \quad i=1,2, \ldots, p, i \neq j ; \\
& 0 \leq L_{j}^{k} \leq z_{j}<\rho_{j}^{k}, \quad i=j,
\end{aligned}
$$

we can get

$$
\begin{aligned}
v\left(Z_{a}^{k}\right)= & \max _{z \in Z_{a}^{k}, y \in \Lambda}\left(\sum_{i=1}^{T} z_{i} \varphi_{i}(y)-\sum_{i=T+1}^{p} z_{i} z_{i} \varphi_{i}(y)\right) \\
\leq & \max _{z \in Z_{a}^{k}, y \in \Lambda} \sum_{i=1, i \neq j}^{T} z_{i} \varphi_{i}(y) \\
& -\min _{z \in Z_{a}^{k}, y \in \Lambda} \sum_{i=T+1}^{p} z_{i} \varphi_{i}(y)+\max _{z \in Z_{a}^{k}, y \in \Lambda} z_{i} \varphi_{i}(y) \\
< & \sum_{i=1, i \neq j}^{T} U_{i}^{k} u_{i}^{0}-\sum_{i=T+1}^{p} L_{i}^{k} l_{i}^{0}+\rho_{j}^{k} u_{j}^{0} \\
= & \operatorname{RUB}^{k}-U_{j}^{k} u_{j}^{0}+\rho_{j}^{k} u_{j}^{0}<\mathrm{LB} .
\end{aligned}
$$

Therefore, there does not exist any global optimum point of $\mathrm{EQ}\left(Z^{k}\right)$ over $Z_{a}^{k}$. 
Similarly, when $j \in\{T+1, T+2, \ldots, p\}$, and for any $y \in \Lambda$ and $z \in Z_{b}^{k}$, since

$$
\begin{aligned}
& 0 \leq l_{i}^{0} \leq \varphi_{i}(y) \leq u_{i}^{0}, \\
& 0 \leq L_{i}^{k} \leq z_{i} \leq U_{i}^{k}, \\
& \quad i=1,2, \ldots, p, i \neq j, \\
& 0 \leq \rho_{j}^{k}<z_{j} \leq U_{j}^{k}, \quad i=j,
\end{aligned}
$$

we have

$$
\begin{aligned}
v\left(Z_{b}^{k}\right)= & \max _{z \in Z_{b}^{k}, y \in \Lambda}\left(\sum_{i=1}^{T} z_{i} \varphi_{i}(y)-\sum_{i=T+1}^{p} z_{i} \varphi_{i}(y)\right) \\
\leq & \max _{z \in Z_{b}^{k}, y \in \Lambda} \sum_{i=1}^{T} z_{i} \varphi_{i}(y) \\
& -\min _{z \in Z_{b}^{k}, y \in \Lambda} \sum_{i=T+1, i \neq j}^{p} z_{i} \varphi_{i}(y)-\min _{z \in Z_{b}^{k}, y \in \Lambda} z_{i} \varphi_{i}(y) \\
< & \sum_{i=1}^{T} U_{i}^{k} u_{i}^{0}-\sum_{i=T+1, i \neq j}^{p} L_{i}^{k} l_{i}^{0}-\rho_{j}^{k} l_{j}^{0} \\
= & \mathrm{RUB}^{k}+L_{j}^{k} l_{j}^{0}-\rho_{j}^{k} l_{j}^{0}<\mathrm{LB} .
\end{aligned}
$$

Therefore, there does not exist any global optimum point of $\mathrm{EQ}\left(Z^{k}\right)$ over $Z_{b}^{k}$.

By the above Theorem 3, we can construct an out space pruning technique to prune a part of the investigated out space region which does not contain the global optimum point of the problem (EQ). Assume that a subbox

$$
Z^{k}=\left(Z_{i}^{k}\right)_{p \times 1} \subseteq Z^{0} \quad \text { with } Z_{i}^{k}=\left[L_{i}^{k}, U_{i}^{k}\right]
$$

will be pruned; then from the Theorem 3 , the investigated box $Z^{k}$ can be renewed by a subbox

$$
\begin{aligned}
& Z^{\prime}=\left(Z_{i}^{\prime}\right)_{p \times 1} \\
& \text { with } Z_{i}^{\prime}= \begin{cases}{\left[\rho_{i}^{k}, U_{i}^{k}\right] \cap Z_{i}^{k},} & i \in\{1,2, \ldots, T\}, \\
{\left[L_{i}^{k}, \rho_{i}^{k}\right] \cap Z_{i}^{k},} & i \in\{T+1, T+2, \ldots, p\} .\end{cases}
\end{aligned}
$$

\section{Algorithm and Its Convergence}

In this section, by utilizing the above new pruning technique, we will present a new out space branch-and-bound algorithm for globally solving the problem (SLRP). In the algorithm, the branching operation is performed in out space $R^{p}$. Assume that $Z=\left\{z \in R^{p} \mid L_{i} \leq z_{i} \leq U_{i}, i=1,2, \ldots, p\right\}$ is $Z^{0}$ or a subbox of it, which will be partitioned, the branching rule is selected as follows.
Set

$$
\begin{aligned}
q \in & \arg \max \left\{U_{i}-L_{i} \mid i=1,2, \ldots, p\right\}, \\
Z^{1} & =\left\{z \in R^{p} \mid L_{i} \leq z_{i} \leq \frac{L_{q}+U_{q}}{2}, i=q, L_{i} \leq z_{i}\right. \\
& \left.\leq U_{i}, i=1,2, \ldots, p, i \neq q\right\}, \\
Z^{2} & =\left\{z \in R^{p} \mid \frac{L_{q}+U_{q}}{2} \leq z_{i} \leq U_{i}, i=q, L_{i} \leq z_{i}\right.
\end{aligned}
$$

$$
\left.\leq U_{i}, i=1,2, \ldots, p, i \neq q\right\} .
$$

Obviously, from [26] we can get that this branching rule is exhaustive; that is, if $\left\{Z^{k}\right\}$ represents a nested subsequence of boxes (i.e., $Z^{k+1} \subseteq Z^{k}$, for all $k$ ) formed by partitioning process, then there exists a unique point $z^{*} \in R^{p}$ satisfying $\bigcap_{k} Z^{k}=\left\{z^{*}\right\}$.

4.1. Out Space Branch-and-Bound Algorithm. Based upon the above linear relaxation bounding problem, the new out space pruning technique, and bisection method, an out space branch-and-bound algorithm is proposed for globally solving the (SLRP) as follows.

Step 1. Let $k=0, \Delta_{0}=\left\{Z^{0}\right\}, \varepsilon \geq 0, l_{i}^{0}=\min _{y \in \Lambda} \varphi_{i}(y)$, and $u_{i}^{0}=\max _{y \in \Lambda} \varphi_{i}(y), i=1,2, \ldots, p$. And let

$$
\begin{gathered}
U_{i}^{0}= \begin{cases}\frac{1}{\min _{y \in \Lambda} \phi_{i}(y)}, & i=1,2, \ldots, T, \\
\frac{1}{-\max _{y \in \Lambda} \phi_{i}(y)}, & i=T+1, \ldots, p,\end{cases} \\
L_{i}^{0}= \begin{cases}\frac{1}{\max _{y \in \Lambda} \phi_{i}(y)}, & i=1,2, \ldots, T, \\
\frac{1}{-\min _{y \in \Lambda} \phi_{i}(y)}, & i=T+1, \ldots, p,\end{cases}
\end{gathered}
$$

and construct the initial box

$$
Z^{0}=\left\{z \in R^{p} \mid L_{i}^{0} \leq z_{i} \leq U_{i}^{0}, i=1,2, \ldots, p\right\} .
$$

Using simplex method to solve $\operatorname{LRP}\left(Z^{0}\right)$, set its optimum solution $y^{0}$ and optimum value $\mathrm{UB}\left(Z^{0}\right)$, respectively. Let $\mathrm{UB}_{0}=\mathrm{UB}\left(Z^{0}\right), z_{i}^{0}=\phi_{i}\left(y^{0}\right)(i=1,2, \ldots, p), \mathrm{LB}_{0}=$ $H_{0}\left(y^{0}, z^{0}\right)$. If $\mathrm{UB}_{0}-\mathrm{LB}_{0} \leq \varepsilon$, then $\left(y^{0}, z^{0}\right)$ and $y^{0}$ are global optimum solutions of $\mathrm{EQ}\left(Z^{0}\right)$ and the (SLRP), respectively. Otherwise, let $F=\emptyset, k=1$ and continue to the following Step 2.

Step 2. Set $\mathrm{LB}=\mathrm{LB}_{k-1}$. For each investigated subbox $Z^{k-1}$, use the former out space pruning technique to prune the investigated subbox $Z^{k-1}$, let the remaining box be $Z^{\prime}$, and set $Z^{k-1}=Z^{\prime}, \mathrm{LB}_{k}=\mathrm{LB}_{k-1}$. 
Step 3. Partition $Z^{k-1}$ into two subboxes $Z^{k, 1}, Z^{k, 2} \subseteq Z^{k-1}$ using the bisection technique. Let the new partitioned subboxes set be $\bar{Z}^{k}$. Set $F=F \cup\left\{Z^{k-1}\right\}$. For each $Z^{k, t} \in \bar{Z}^{k}$, solve the (LRP) using simplex method to get $\operatorname{UB}\left(Z^{k, t}\right)$ and $y^{k, t}$, and set $z_{i}^{k, t}=\phi_{i}\left(y^{k, t}\right), i=1,2, \ldots, p$. If $\mathrm{UB}\left(Z^{k, t}\right)<\mathrm{LB}_{k}$, then let $\bar{Z}^{k}:=\bar{Z}^{k} \backslash Z^{k, t}, F=F \cup\left\{Z^{k, t}\right\}$; otherwise, update $\mathrm{LB}_{k}=\min \left\{\mathrm{LB}_{k}, H_{0}\left(y^{k, t}, z^{k, t}\right)\right\}$, if necessary.

Step 4. Let the remaining partitioned set $\Delta_{k-1}:=\left(\Delta_{k-1} \mid\right.$ $\left.Z^{k-1}\right) \cup\left\{\bar{Z}^{k}\right\}$, and let the new upper bound $\mathrm{UB}_{k}=$ $\max _{Z \in \Delta_{k-1}} \mathrm{UB}(Z)$. Let $\Delta_{k}=\left\{Z \mid Z \in\left(\Delta_{k-1} \cup\left\{Z^{k, 1}, Z^{k, 2}\right\}\right), Z \notin\right.$ $F\}$ and $\mathrm{UB}_{k}=\min \left\{\mathrm{UB}(Z) \mid Z \in \Delta_{k}\right\}$. If $\mathrm{UB}_{k}-\mathrm{LB}_{k} \leq \varepsilon$, then we get that $\left(y^{k}, z^{k}\right)$ and $y^{k}$ are global optimum points of the $\mathrm{EQ}\left(Z^{0}\right)$ and the (SLRP), respectively. Otherwise, let $k=k+1$ and return to Step 2.

4.2. Convergence Analysis. In the following, we describe the global convergence of the above algorithm.

Theorem 4. The above algorithm either stops finitely to obtain the global optimum point of the (SLRP) or produces an infinite sequence $\left\{y^{k}\right\}$ whose accumulation point $y^{*}$ must be the global optimum point of the (SLRP).

Proof. If the proposed algorithm stops finitely at $k_{\text {th }}$ iteration, where $k \geq 0$, obviously, when the algorithm stops, we can get $y^{k}$ and $\left(y^{k}, z^{k}\right)$ by solving the $\operatorname{LRP}\left(Z^{k}\right)$, which are the feasible points of the (SLRP) and the (EQ), where

$$
\begin{aligned}
& z_{i}^{k}=\frac{1}{\phi_{i}\left(y^{k}\right)}, \quad i=1,2, \ldots, T \\
& z_{i}^{k}=\frac{1}{-\phi_{i}\left(y^{k}\right)}, \quad i=T+1, T+2, \ldots, p
\end{aligned}
$$

and by terminating step of the algorithm, the updating operations of the lower bound and upper bound, and Theorems 1 and 2 , we can easily follow that

$$
\begin{aligned}
H_{0}\left(y^{k}, z^{k}\right) & \geq \mathrm{UB}_{k}-\varepsilon, \\
\mathrm{UB}_{k} & \geq v, \\
v & \geq H_{0}\left(y^{k}, z^{k}\right), \\
G\left(y^{k}\right) & =H_{0}\left(y^{k}, z^{k}\right) .
\end{aligned}
$$

By combining the above all inequalities and equality, we can easily get that

$$
v-\epsilon \leq G\left(y^{k}\right) \leq v
$$

Therefore, if the algorithm stops finitely at $k_{\text {th }}$ iteration, then $y^{k}$ is the global $\epsilon$-optimal solution of the (SLRP).
If the above algorithm generates an infinite sequence $\left\{y^{k}\right\}$ of incumbent solutions by solving the $\operatorname{LRP}\left(Z^{k}\right)$, let

$$
\begin{aligned}
& z_{i}^{k}=\frac{1}{\phi_{i}\left(y^{k}\right)}, \quad i=1,2, \ldots, T \\
& z_{i}^{k}=\frac{1}{-\phi_{i}\left(y^{k}\right)}, \quad i=T+1, T+2, \ldots, p
\end{aligned}
$$

then we can get an infinite sequence $\left\{y^{k}, z^{k}\right\}$ of incumbent solutions for $\operatorname{EQ}\left(Z^{k}\right)$. By the continuity character of the function $\phi_{i}\left(y^{k}\right)$ and $1 / \phi_{i}\left(y^{k}\right)=z_{i}^{k} \in\left[L_{i}^{k}, U_{i}^{k}\right](i=1, \ldots, T)$ and $1 /-\phi_{i}\left(y^{k}\right)=z_{i}^{k} \in\left[L_{i}^{k}, U_{i}^{k}\right](i=T+1, \ldots, p)$, and the exhaustiveness of the bisection rule, we can get the following conclusions, for each $i \in\{1,2, \ldots, T\}$ :

$$
\begin{aligned}
\frac{1}{\phi_{i}\left(y^{*}\right)} & =\lim _{k \rightarrow \infty} \frac{1}{\phi_{i}\left(y^{k}\right)}=\lim _{k \rightarrow \infty} L_{i}^{k}=\lim _{k \rightarrow \infty} U_{i}^{k} \\
& =\lim _{k \rightarrow \infty}\left[L_{i}^{k}, U_{i}^{k}\right]=\bigcap_{k}\left[L_{i}^{k}, U_{i}^{k}\right]=\left\{z_{i}^{*}\right\} ;
\end{aligned}
$$

for each $i \in\{T+1, T+2, \ldots, p\}$,

$$
\begin{aligned}
\frac{1}{-\phi_{i}\left(y^{*}\right)} & =\lim _{k \rightarrow \infty} \frac{1}{-\phi_{i}\left(y^{k}\right)}=\lim _{k \rightarrow \infty} L_{i}^{k}=\lim _{k \rightarrow \infty} U_{i}^{k} \\
& =\lim _{k \rightarrow \infty}\left[L_{i}^{k}, U_{i}^{k}\right]=\bigcap_{k}\left[L_{i}^{k}, U_{i}^{k}\right]=\left\{z_{i}^{*}\right\} .
\end{aligned}
$$

Therefore, $\left(y^{*}, z^{*}\right)$ is a feasible point of the $\operatorname{EQ}\left(Z^{0}\right)$, also since $\left\{\mathrm{UB}\left(Z^{k}\right)\right\}$ is a decreasing real number sequence bounded by $v$, we can follow that

$$
\begin{aligned}
H_{0}\left(y^{*}, z^{*}\right) & \leq v \leq \lim _{k \rightarrow \infty} \operatorname{UB}\left(Z^{k}\right) \\
& =\lim _{k \rightarrow \infty}\left(\sum_{i=1}^{T} U_{i}^{k} \varphi_{i}\left(y^{k}\right)-\sum_{i=T+1}^{p} L_{i}^{k} \varphi_{i}\left(y^{k}\right)\right) \\
& =H_{0}\left(y^{*}, z^{*}\right) .
\end{aligned}
$$

Thus, from the updating method of the lower bound and the continuity character of the $G(y)$, we can get that

$$
\begin{aligned}
\lim _{k \rightarrow \infty} \operatorname{LB}\left(Z^{k}\right) & =v=H_{0}\left(y^{*}, z^{*}\right)=G\left(y^{*}\right) \\
& =\lim _{k \rightarrow \infty} G\left(y^{k}\right)=\lim _{k \rightarrow \infty} \operatorname{UB}\left(Z^{k}\right) .
\end{aligned}
$$

Hence, $y^{*}$ is the global optimum point of the (SLRP); the conclusion is proved.

4.3. Numerical Example. In this subsection, some wellknown test problems are implemented on Intel(R) Core(TM)2 Duo CPU microcomputer to verify the performance of the proposed out space branch-and-bound algorithm. The proposed out space branch-and-bound algorithm is coded in $\mathrm{C}++$ program, and the simplex method is used to solve each linear relaxation programming problem. 
TABLE 1: Numerical results for test problem.

\begin{tabular}{|c|c|c|c|c|c|c|}
\hline Number & $(m, n, p, c)$ & Ref. & Ave. Iter. & Ave. L. & Ave. Time (s) & $\epsilon$ \\
\hline \multirow{3}{*}{ (1) } & $(2,3,3,2)$ & This paper & 3.2 & 3.1 & 0.015 & $10^{-6}$ \\
\hline & $(2,3,3,2)$ & [15] & 38.3 & 7.8 & 0.05 & $10^{-6}$ \\
\hline & $(2,3,3,2)$ & {$[16]$} & 4.6 & 1.0 & 0 & $10^{-5}$ \\
\hline \multirow{3}{*}{ (2) } & $(4,3,4,2)$ & This paper & 5.2 & 4.6 & 0.05 & $10^{-6}$ \\
\hline & $(4,3,4,2)$ & [15] & 46 & 8.2 & 0.1 & $10^{-6}$ \\
\hline & $(4,3,4,2)$ & {$[16]$} & 5.6 & 1.1 & 0 & $10^{-5}$ \\
\hline \multirow{3}{*}{ (3) } & $(5,10,3,2)$ & This paper & 6.2 & 3.8 & 0.073 & $10^{-6}$ \\
\hline & $(5,10,3,2)$ & [15] & 414.3 & 60.2 & 1.9 & $10^{-6}$ \\
\hline & $(5,10,3,2)$ & {$[16]$} & 6.3 & 1.4 & 0 & $10^{-5}$ \\
\hline \multirow{3}{*}{ (4) } & $(10,20,3,2)$ & This paper & 7.3 & 4.4 & 0.087 & $10^{-6}$ \\
\hline & $(10,20,3,2)$ & [15] & 402.7 & 56.8 & 10.1 & $10^{-6}$ \\
\hline & $(10,20,3,2)$ & {$[16]$} & 7.4 & 2.0 & 0.023 & $10^{-5}$ \\
\hline \multirow{3}{*}{ (5) } & $(15,30,3,2)$ & This paper & 6.5 & 4.5 & 0.130 & $10^{-6}$ \\
\hline & $(15,30,3,2)$ & [15] & 424.3 & 61.6 & 35.1 & $10^{-6}$ \\
\hline & $(15,30,3,2)$ & {$[16]$} & 7.0 & 2.0 & 3.1 & $10^{-5}$ \\
\hline
\end{tabular}

Numerical problems and their computational results are summarized in Table 1. Some notations have been also used for column headers in Table 1 as follows. Ave. Iter. represents the average number of iterations of the algorithm; Ave. L. represents the average number of the necessary maximum nodes of the algorithm; Ave. Time (s) stands for the execution time of algorithm in seconds.

Problem (see $[15,16])$.

$$
\begin{array}{ll}
\max & \sum_{j=1}^{p} \frac{\sum_{i=1}^{n} d_{j i} y_{i}+c}{\sum_{i=1}^{n} \sigma_{j i} y_{i}+c} \\
\text { s.t. } & \sum_{i=1}^{n} a_{k i} y_{i} \leq b_{k}, \quad k=1, \ldots, m, \\
& y_{i} \geq 0.0, \quad i=1,2, \ldots, n,
\end{array}
$$

where $d_{j i}, \sigma_{j i}, a_{k i}, j=1, \ldots, p, k=1, \ldots, m, i=1, \ldots, n$, are randomly generated in $[0.0,1.0] ; c$ are generated in $[1.0,100.0] ; b_{k}=1.0, k=1,2, \ldots, m$. In the investigated problem, $m$ denotes the number of the constraints, $n$ denotes the dimension of the problem, and $p$ denotes the number of ratios.

From Table 1, compared with the known algorithms, numerical results indicate that the proposed algorithm can be used to globally solve the problems (SLRP) with the higher computational efficiency.

\section{Concluding Remarks}

In this paper, we present an effective algorithm for globally solving the sum of linear ratios problem (SLRP). By introducing new variables, we transform the initial sum of linear ratios problems (SLRP) into the equivalent nonconvex programming problem. Next, the linear relaxation programming problem of the equivalent problem is established by utilizing direct relaxation. To improve the computational efficiency of the algorithm, an out space pruning technique is derived, which offers a possibility of pruning a large part of the investigated out space region which does not contain the optimal solution of the equivalent problem. Based on out space partition, by combining bounding technique and reduction technique, a new outcome space branch-andbound algorithm for globally solving the sum of linear ratios problem (SLRP) is constructed. Finally, numerical problems and their computational results are presented to demonstrate both computational efficiency and solution quality of the proposed algorithm.

In future research, we can extend the proposed algorithm and reduction technique to solve nonlinear fractional problems, like concave-convex sum-of-ratios problem, convexconvex sum-of-ratios problem, linear fractional multiplicative problem, generalized linear fractional programming problem, and so on.

\section{Conflicts of Interest}

The authors declare that they have no conflicts of interest.

\section{Acknowledgments}

This paper is supported by the Cultivation Plan of Young Key Teachers in Colleges and Universities of Henan Province (2016GGJS-107), the Basic and Advanced Technology Research Project of Henan Province (152300410097), the Key Scientific Research Project of Universities in Henan Province (17B413002, 16A110014, and 17A110021), the Major Scientific Research Projects of Henan Institute of Science and Technology (2015ZD07), the National Natural Science Foundation of Henan Province (152300410097), and Science and Technology Project of Henan Province (162102210294). 


\section{References}

[1] Y. Almogy and O. Levin, Parametric Analysis of a Multistage Stochastic Shipping Problem, Operational Research 69, Tavistock Publications, London, England, UK, 1970.

[2] H. P. Benson, "A simplicial branch and bound duality-bounds algorithm for the linear sum-of-ratios problem," European Journal of Operational Research, vol. 182, no. 2, pp. 597-611, 2007.

[3] C. S. Colantoni, R. P. Manes, and A. Whinston, "Programming, profit rates, and pricing decisions, accounting review," vol. 44, pp. 467-481, 1969.

[4] M. R. Rao, "Cluster analysis and mathematical programming," Journal of the American Statistical Association, vol. 66, no. 335, pp. 622-626, 1971.

[5] H. Konno and H. Watanabe, "Bond portfolio optimization problems and their applications to index tracking: a partial optimization approach," Journal of the Operations Research Society of Japan, vol. 39, no. 3, pp. 295-306, 1996.

[6] A. Charnes and W. W. Cooper, "Programming with linear fractional functionals," Naval Research Logistics Quarterly, vol. 9, pp. 181-186, 1962.

[7] H. Konno, Y. Yajima, and T. Matsui, "Parametric simplex algorithms for solving a special class of nonconvex minimization problem," Journal of Global Optimization, vol. 1, pp. 65-81, 1991.

[8] J. E. Falk and S. W. Palocsay, "Image space analysis of generalized fractional programs," Journal of Global Optimization, vol. 4, pp. 63-88, 1994.

[9] Y. Ji, K.-C. Zhang, and S.-J. Qu, "A deterministic global optimization algorithm," Applied Mathematics and Computation, vol. 185, no. 1, pp. 382-387, 2007.

[10] H. Konno and K. Fukaishi, "A branch and bound algorithm for solving low rank linear multiplicative and fractional programming problems," Journal of Global Optimization, vol. 18, pp. 283299, 2000.

[11] P.-P. Shen and C.-F. Wang, "Global optimization for sum of linear ratios problem with coefficients," Applied Mathematics and Computation, vol. 176, no. 1, pp. 219-229, 2006.

[12] C.-F. Wang and P.-P. Shen, "A global optimization algorithm for linear fractional programming," Applied Mathematics and Computation, vol. 204, no. 1, pp. 281-287, 2008.

[13] H. Jiao, "A branch and bound algorithm for globally solving a class of nonconvex programming problems," Nonlinear Analysis, vol. 70, no. 2, pp. 1113-1123, 2009.

[14] H. P. Benson, "On the global optimization of sums of linear fractional functions over a convex set," Journal of Optimization Theory and Applications, vol. 121, no. 1, pp. 19-39, 2004.

[15] W. Yanjun, S. Peiping, and L. Zhian, "A branch-and-bound algorithm to globally solve the sum of several linear ratios," Applied Mathematics and Computation, vol. 168, no. 1, pp. 89101, 2005.

[16] Y. Shi, Global optimization for sum of ratios problems, Dissertation of master degree for Henan Normal University, Henan Normal University, 2011.

[17] N. T. H. Phuong and H. Tuy, "A unified monotonic approach to generalized linear fractional programming," Journal of Global Optimization, vol. 26, no. 3, pp. 229-259, 2003.

[18] H.-W. Jiao and S.-Y. Liu, "A practicable branch and bound algorithm for sum of linear ratios problem," European Journal of Operational Research, vol. 243, no. 3, pp. 723-730, 2015.

[19] H. Jiao, S. Liu, J. Yin, and Y. Zhao, "Outcome space range reduction method for global optimization of sum of affine ratios problem," Open Mathematics, vol. 14, pp. 736-746, 2016.
[20] H. Jiao, C. Bai, and X. Wang, "Reduction-branch-bound algorithm for solving sum of linear ratios problems," Mathematica Applicata, vol. 29, no. 3, pp. 625-631, 2016.

[21] H.-W. Jiao, S.-Y. Liu, and Y.-F. Zhao, "Effective algorithm for solving the generalized linear multiplicative problem with generalized polynomial constraints," Applied Mathematical Modelling. Simulation and Computation for Engineering and Environmental Systems, vol. 39, no. 23-24, pp. 7568-7582, 2015.

[22] B. L. Gorissen, "Robust fractional programming," Journal of Optimization Theory and Applications, vol. 166, no. 2, pp. 508528, 2015.

[23] A. Aubry, V. Carotenuto, and A. De Maio, "New Results on Generalized Fractional Programming Problems With Toeplitz Quadratics," IEEE Signal Processing Letters, vol. 23, no. 6, pp. 848-852, 2016.

[24] N. Ruan and D. Y. Gao, "Global solutions to fractional programming problem with ratio of nonconvex functions," Applied Mathematics and Computation, vol. 255, pp. 66-72, 2015.

[25] H. Li, "A genetic algorithm using a finite search space for solving nonlinear/linear fractional bilevel programming problems," Annals of Operations Research, vol. 235, pp. 543-558, 2015.

[26] R. Horst and H. Tuy, Global Optimization: Deterministic Approaches, Springer, Berline, Germany, 2nd edition, 1993. 


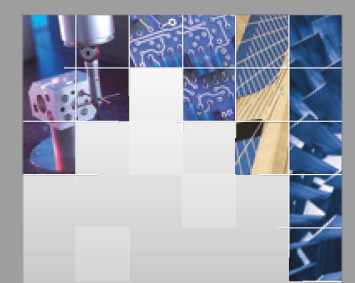

\section{Enfincering}
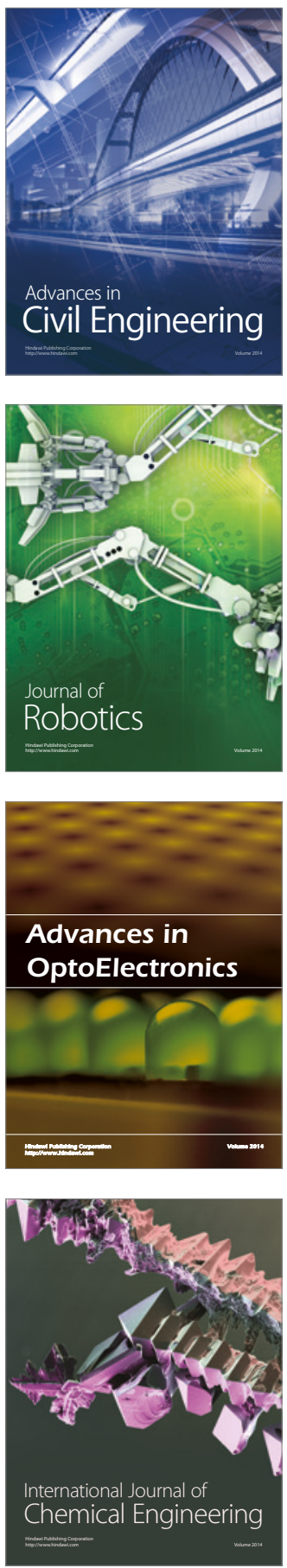

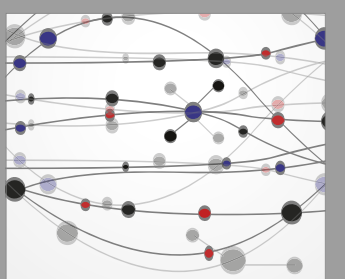

The Scientific World Journal

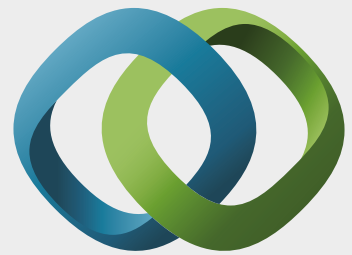

\section{Hindawi}

Submit your manuscripts at

https://www.hindawi.com
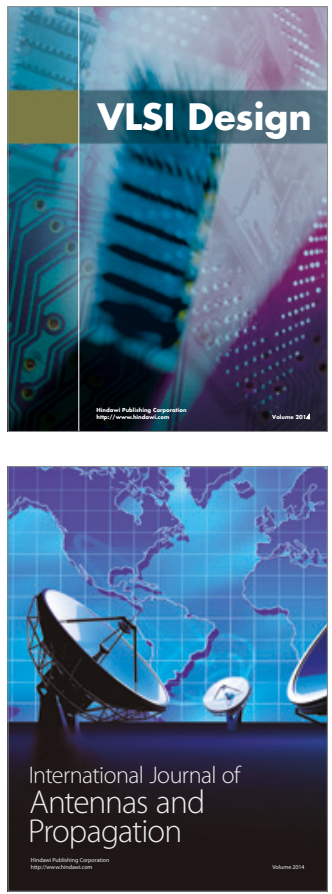

\section{Rotating}

Machinery
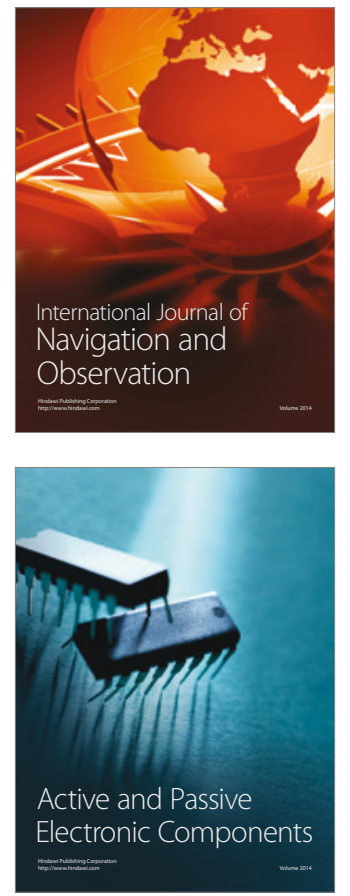
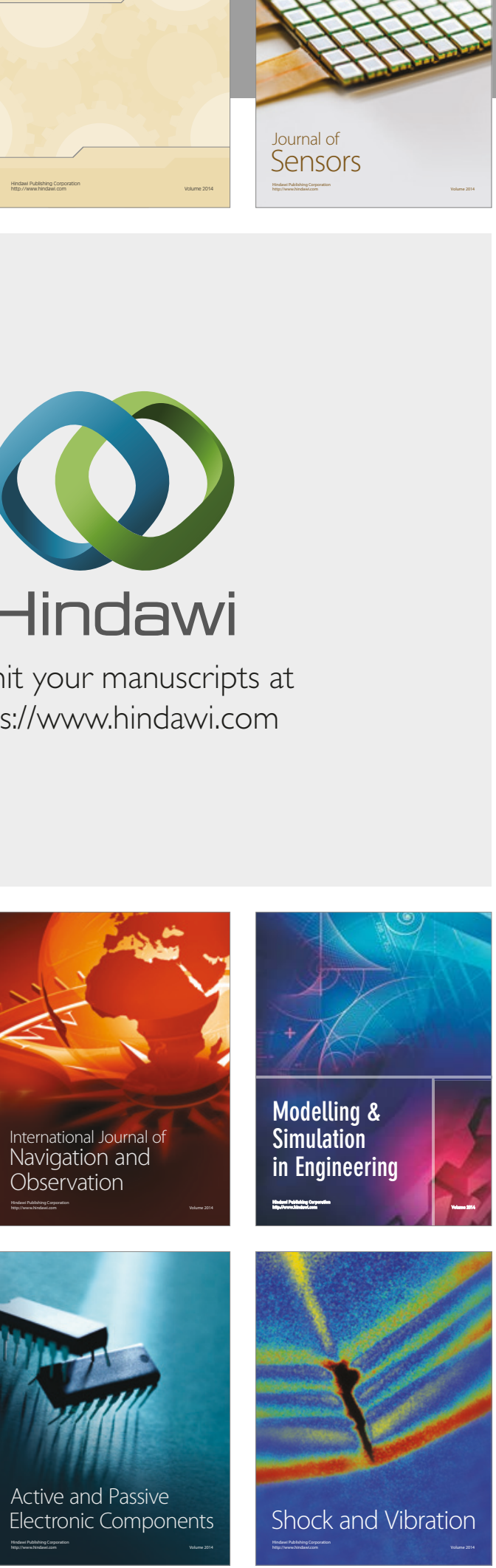
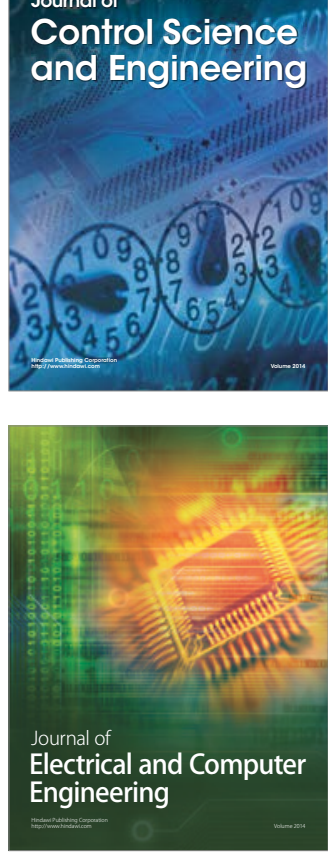

Distributed

Journal of

Control Science

and Engineering
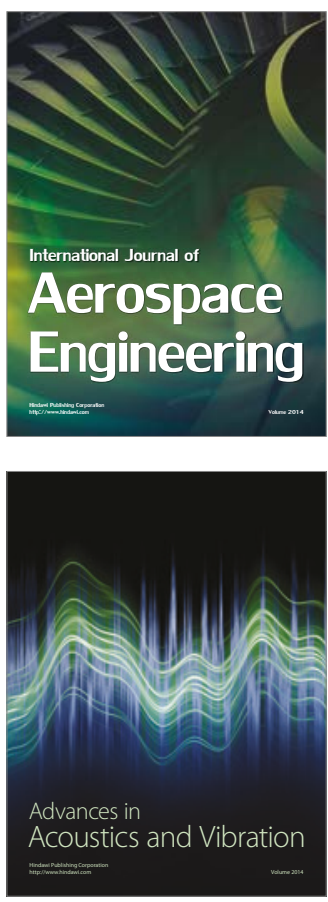

Sensor Networks 